\title{
Skill reconsolidation does not operate at a rapid micro-timescale level
}

Jasmine Herszage $^{1}$, Marlene Bönstrup ${ }^{2}$, Leonardo G Cohen ${ }^{3}$ and Nitzan Censor ${ }^{1}$

1. School of Psychological Sciences and Sagol School of Neuroscience, Tel Aviv University, Tel Aviv 69978, Israel

2. Department of Neurology, University of Leipzig, Germany

3. Human Cortical Physiology and Neurorehabilitation Section, NINDS, NIH, Bethesda, MD, USA

\begin{abstract}
Abundant evidence shows that consolidated memories are susceptible to modifications following their reactivation through reconsolidation. Processes of memory consolidation and reconsolidation have been commonly documented after hours or days. Motivated by studies showing rapid consolidation in early stages of skill acquisition, here we asked whether skill memories are susceptible to modifications through rapid reconsolidation, even at initial stages of learning. In a set of experiments, we collected crowdsourced online motor sequence data to test whether post-reactivation interference and enhancement occur through rapid reconsolidation. Results indicate that memories forming during early learning are not susceptible to interference nor to enhancement within a rapid reconsolidation time window, relative to control conditions. This set of evidence suggests that memory reconsolidation might be dependent on consolidation at the macro-timescale level, requiring hours or days to occur.
\end{abstract}




\section{Introduction}

Consolidation is a crucial process in the formation of memories, occurring after the initial encoding of information or a skill, and resulting in memory stabilization. While previously thought to be an irreversible process $(1,2)$, evidence from studies in rodents (3-5), further supported by human studies (6-8) indicates that even fully consolidated memories can become unstable again upon their reactivation. Such reactivation can result in deterioration (7-10) or in enhancement of the memory through reconsolidation ((11), for a review see (12)). Similar to previous reports across multiple memory domains $(13,14)$, spanning from fear memory $((3,4$, 15) to perceptual memories (11), evidence for such modification of memories during reconsolidation was recently shown in motor skill memories as well: reactivations were shown to protect memories from future interference (16) and even induce learning of a motor skill (17, $18)$.

At the neural level, reconsolidation is known as a protein synthesis-dependent process (3), therefore requiring hours or days to occur (19-21). While similar timescales were reported for consolidation as well (22-25), it has been shown that a rapid form of consolidation occurs even in early stages of learning, at a micro-timescale of minutes (26). Thus, while consolidated memories often show offline learning gains between sessions, such gains were evident between trials in early stages of a single encoding session, at a micro-timescale level.

Motivated by this set of evidence, we asked whether reactivated skill memories would be susceptible to modifications through rapid 'micro-timescale reconsolidation'. In a set of experiments, we collected crowdsourced motor sequence learning (27) data from 459 participants recruited from the Amazon Mechanical Turk platform (MTurk), to test whether postreactivation interference and enhancement occur through rapid micro-timescale reconsolidation, according to the established human reconsolidation criteria (14). Since memories were shown to be susceptible to interference following reactivation $(8,28)$, Experiment 1 tested whether memories following rapid consolidation are susceptible to interference within a rapid reconsolidation time window. Participants underwent rapid micro-timescale consolidation (26) of the motor sequence task. Then, an interfering sequence was presented interleaved with brief reactivations of the original sequence. Retest was compared to a control group without reactivations (see figure 1b). Since reactivations were recently shown to induce learning of motor 
skills through reconsolidation (17), Experiment 2 tested if memories following rapid consolidation can benefit from rapid reconsolidation to produce enhanced learning gains. Following micro-timescale consolidation, the memory was reactivated repeatedly to induce learning. Retest was compared to a control group without reactivations (see figure 1c).

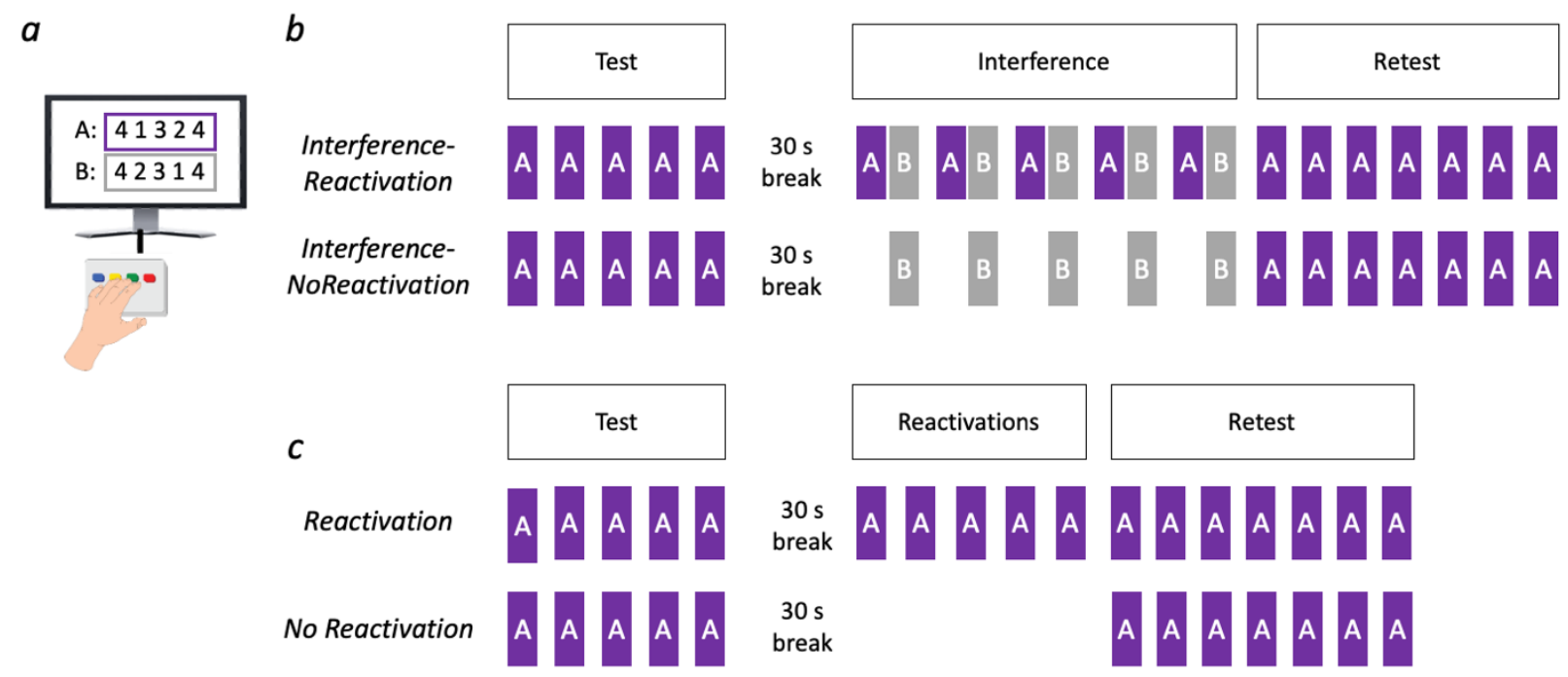

Figure 1: Experimental design. (a) The sequence tapping task required participants to tap a sequence of numbers (either sequence A: 41324 or sequence B: 423 14) which was constantly presented on the screen during trials. (b) Experimental design of Experiment 1. Each purple square represents a trial of the main sequence A, and each grey square represents a trial of the new sequence B. (c) Experimental design of Experiment 2.

\section{Materials and Methods}

\section{Participants}

Participants were recruited from the Amazon Mechanical Turk Platform (MTurk). Qualifications for registered MTurk workers to participate in the experiments were: $>95 \%$ approval rate on all previous MTurk assignments, location in the United States, right-handedness and no previous participation in any sequence learning task offered by our lab. All procedures were in accordance with a protocol approved by the Tel Aviv University Ethics committee. All participants declared via button press that they agree to participate and acknowledge the outline and purpose of the study, their voluntary participation, the time commitment and payment, their mandatory age above 18 years old, information on data safety and being given contact information. 
Sample sizes for each experiment were estimated based on power analysis of pilot studies of motor sequence learning. Sample sizes were 230 participants for Experiment 1 ( 95 female, mean \pm sd age $33.9 \pm 7.2$ ), and 229 for Experiment 2 (110 female, age $34.7 \pm 8.8$ ). These sample sizes represent the total number of participants after exclusion of assignments demonstrating incomplete adherence to task instructions. Participants were paid $1.5 \$$ for the session, equivalent to $>8 \$$ per hour. The time of the task being posted was midday on weekdays for each experiment. Tasks were posted at batches of 30-50 workers each time for administrative (monetary) reasons.

\section{Task}

Participants practiced the motor skill learning task (29) in which they were asked to tap, as fast and as accurate as they could, a five-digit sequence (Experiment 1: main sequence 4-1-3-2-4, new sequence 4-2-3-1-4; Experiment 2: 4-1-3-2-4; see figure 1a) on the numeric keys of participants' computer keyboards with the pinky finger corresponding to button \# 1 , the ring finger to \# 2, middle finger to \# 3 , and index finger to \# 4. During each trial, the sequence was presented constantly on a computer screen. The task was performed with the left nondominant hand in all trials. Each trial lasted 10 seconds, during which feedback was provided in the form of a star displayed at the top portion of the screen, appearing immediately after each keypress regardless of correctness $(17,26)$. Stimuli were programmed, presented and responses recorded using the Pavlovia.org platform.

\section{Experimental Procedure}

All subjects performed an identical encoding session consisting of 5 trials lasting $10 \mathrm{~s}$ each with $10 \mathrm{~s}$ breaks in between. Experiment 1 tested whether early learning memories are susceptible to interference within a rapid reconsolidation time window. Accordingly, the encoding session was followed by a $30 \mathrm{~s}$ break, during which a countdown was consistently presented on the screen to maintain subjects' engagement with the task. Following the break, subjects in the Interference Reactivations group ( $\mathrm{N}=115)$ performed 5 reactivations trials, in which they performed the main sequence (4-1-3-2-4), each immediately followed by a trial of the new sequence (4-2-3-1-4, 5 new sequence trials in total), while subjects in the Interference-NoReactivations group ( $\mathrm{N}=115)$ 
performed the 5 new sequence trials with $10 \mathrm{~s}$ breaks in between, without reactivations. Immediately following these trials, all participants completed a retest session consisting of 7 trials of the main sequence (see figure 1b). Experiment 2 tested if early learning memories can benefit from micro-timescale reconsolidation to produce enhanced learning gains. Accordingly, participants in the Reactivations group $(\mathrm{N}=127)$ performed 5 reactivations trials following the 30 $\mathrm{s}$ break and encoding session, followed by a retest session identical to experiment 1 , while participants in the NoReactivations group $(\mathrm{N}=102)$ performed the encoding and retest sessions without reactivations, and with a break of the same length as the practice of the Reactivations group (120 s; see figure $1 \mathrm{~b})$.

\section{Data analysis}

Behavioral data were analyzed with Matlab R2019b and SPSS statistics 27. Due to the experimental setting offered via crowdsourcing platforms, data registered from each participant were checked for correct implementation and adherence to task instructions. Incorrect implementation of instructions or adherence was defined by (i) completion of the task with the right hand (documented by given answer to question after the task), (ii) completion of only one sequence repetition except for trial 1 (in both experiments) and reactivation trials in Experiment 1, (iii) keypresses consistently different from instructed sequence, (iv) response times until the first key tap longer than 2 seconds, indicative of lack of attention to the screen, (v) no registered responses during at least one trial. Subjects with incorrect implementation (according to at least one of the above) were excluded from analyses.

To test for baseline differences between groups, a repeated measures ANOVA was conducted with the 5 test trials as a within subject factor, and group as a between subject factor. Test-retest improvements were evaluated with a repeated measures ANOVA with 2 performance levels (mean 5 test trials, mean 7 retest trials), and group as a between subject factor, Bonferroni corrected. In experiment 1 , to test for gradual improvements in the new sequence, as well as gradual decrease in performance of the original sequence during reactivations, two repeated measures ANOVA were conducted with 5 trials ( 5 new sequence trials, or 5 reactivation trials) as a within subject factor, Bonferroni corrected. Each null result in an ANOVA test was further confirmed with a Bayesian approach, by calculating the Bayes factor of comparing total learning across the two conditions. Bayesian analyses were performed with JASP version 0.16. 


\section{Results}

To test whether memories express increased susceptibility to interference during early learning within micro-timescale reconsolidation time windows, Experiment 1 compared the effect of interference in motor sequence learning between two groups who experienced interference either with or without reactivations. Both Interference-Reactivation and Interference-NoReactivation groups first performed 5 trials of skill acquisition. A repeated measures ANOVA with 5 test trials and 2 group levels showed no baseline differences between the groups (no group effect $\mathrm{F}_{1,228}=0.07, \mathrm{p}=0.79$ and no group $\mathrm{x}$ trial interaction $\mathrm{F}_{4,912}=0.80, \mathrm{p}=0.52$; see figure $2 \mathrm{a}$ ), with a complimentary Bayesian analysis confirming that there was substantial support in favor of the null hypothesis of no difference between groups relative to the alternative hypothesis for the group effect $\left(\mathrm{BF}_{01}=7.10\right)$ and the group $\mathrm{x}$ trial interaction $\left(\mathrm{BF}_{01}=127.31\right)$. Test-retest improvements showed a main effect for time $\left(\mathrm{F}_{1,228}=207.69\right.$, $\left.\mathrm{p}<0.001\right)$, but no effect for group $\left(\mathrm{F}_{1,228}=0.11, \mathrm{p}=0.74 ; \mathrm{BF}_{01}=3.17\right)$ nor a significant interaction $\left(\mathrm{F}_{1,228}=3.40, \mathrm{p}=0.07\right.$; see figure $2 \mathrm{~b}$ c), suggesting that micro-timescale reconsolidation time windows did not modulate the susceptibility to interference at early stage of skill learning. Of note, while retest performance was not significantly different between groups $\left(\mathrm{F}_{1,228}=0.76, \mathrm{p}=0.39\right)$, post-hoc pairwise comparisons showed that the first retest trial was better in the Interference-Reactivation group, compared to the Interference-NoReactivation group $\left(\mathrm{t}_{228}=2.96, \mathrm{p}=0.003\right)$. However, this difference diminished in the following retest trials and was not significant in any of the following retest trials (significance level: $\mathrm{p}=0.37, \mathrm{p}=0.97, \mathrm{p}=0.53, \mathrm{p}=0.95, \mathrm{p}=0.64, \mathrm{p}=0.58$ for trials $2-7$ respectively; Bonferroni corrected). 

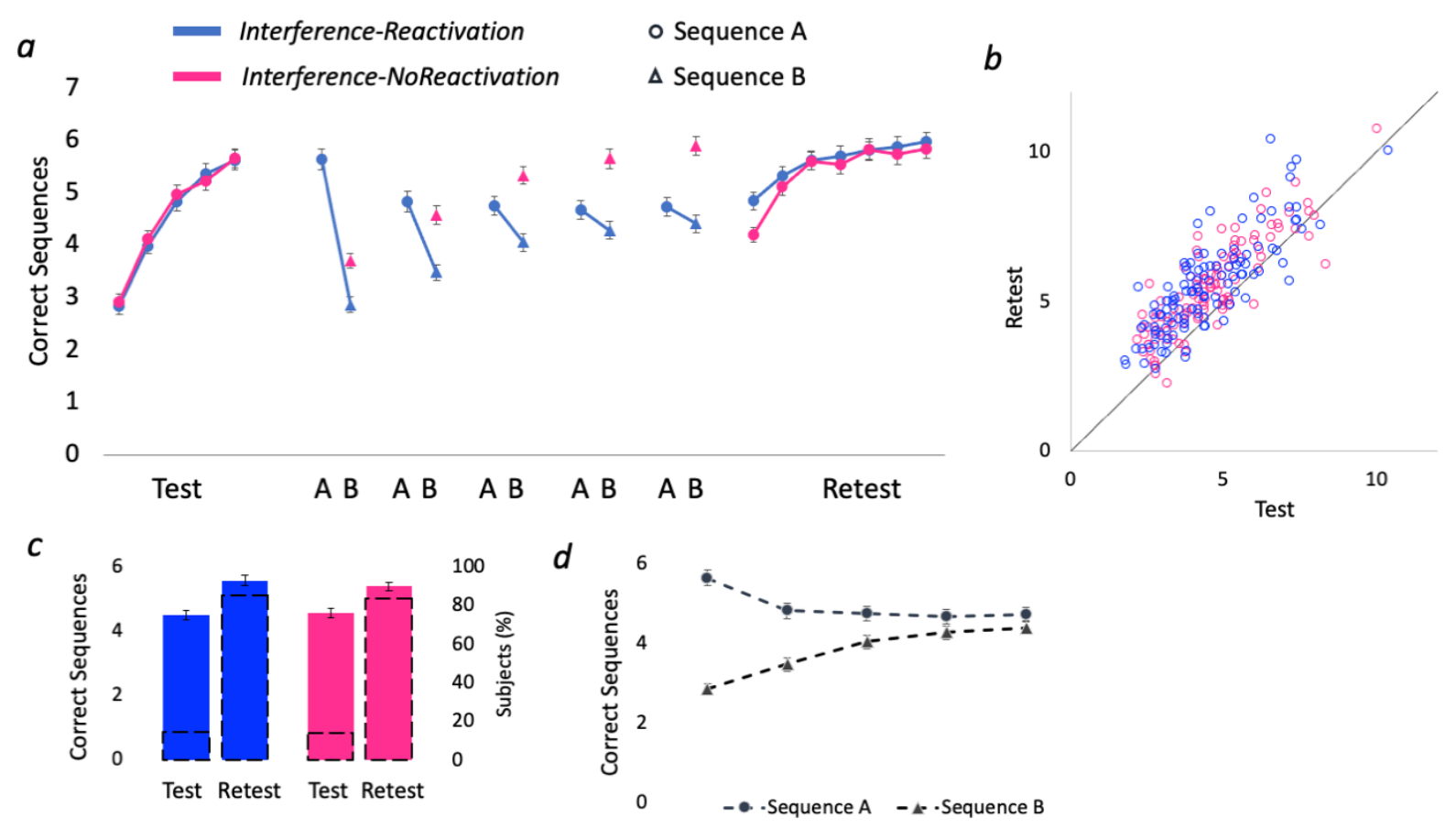

Figure 2: Micro-timescale reactivations do not enhance memory interference. (a) Single-trial performance (Interference-Reactivations group in blue and Interference-NoReactivations group in pink). (b) Test versus retest single-subject performance presented in a scatterplot along a unit slope line $(\mathrm{y}=\mathrm{x})$ where each point reflects a participant $(11,16,30)$. Data accumulating above the unit line reflect subjects who improved from test to retest, expressing less interference, while data points below the line indicate degraded retest performance, expressing stronger interference. (c) Colored bars reflect the mean performance in test and retest sessions (corresponding to the left y-axis), dashed black bars (corresponding to the right y-axis) reflect the percentage of participants on each side of the unit slope line in b. (d) Mean performance of the Interference-Reactivations group in both sequences executed alternatingly (main sequence A as circles, interfering sequence B as triangles). Error bars represent SEM.

Of note, while performance in trials of the new memory showed gradual improvements $\left(\mathrm{F}_{4,456}=40.42, \mathrm{p}<0.001\right.$, Greenhouse-Geisser corrected; see triangles in figure $\left.2 \mathrm{~d}\right)$, performance in the reactivation trials showed gradual decrease $\left(\mathrm{F}_{4,456}=12.54, \mathrm{p}<0.001\right.$, Greenhouse-Geisser corrected; see circles in figure 2d), suggesting that in early stages of learning, two memories can be encoded in parallel, converging performance to a shared similar level.

Experiment 2 tested if early learning memories can benefit from micro-timescale reconsolidation and produce enhanced learning gains. To further investigate whether reconsolidation operates at a micro-timescale level, Experiment 2 tested whether such immediate reactivations of the memory induce learning, as recently reported between-days (17). Thus, Experiment 2 maintained 
similar test and retest as in Experiment 1, with either 5 reactivations trials (Reactivation group) between test and retest, or without reactivations (NoReactivation group). A repeated measures ANOVA with 5 test trials and 2 group levels showed comparable baseline performance across groups during acquisition and test trials (no group effect $\mathrm{F}_{1,227}=1.16, \mathrm{p}=0.28, \mathrm{BF}_{01}=4.16$ and no group $\mathrm{x}$ trial interaction $\mathrm{F}_{4,908}=0.44, \mathrm{p}=0.78, \mathrm{BF}_{01}=155.20$; see figure $3 \mathrm{a}$ ). Test-retest improvements showed a main effect for time $\left(\mathrm{F}_{1,227}=538.91, \mathrm{p}<0.001\right)$, but no effect for group $\left(\mathrm{F}_{1,227}=1.42, \mathrm{p}=0.24, \mathrm{BF}_{01}=2.51\right)$ nor a significant interaction $\left(\mathrm{F}_{1,227}=0.10, \mathrm{p}=0.76, \mathrm{BF}_{01}=4.71\right.$; see figure $3 \mathrm{~b}-\mathrm{c}$ ), suggesting that micro-timescale reconsolidation time windows did not induce learning gains.
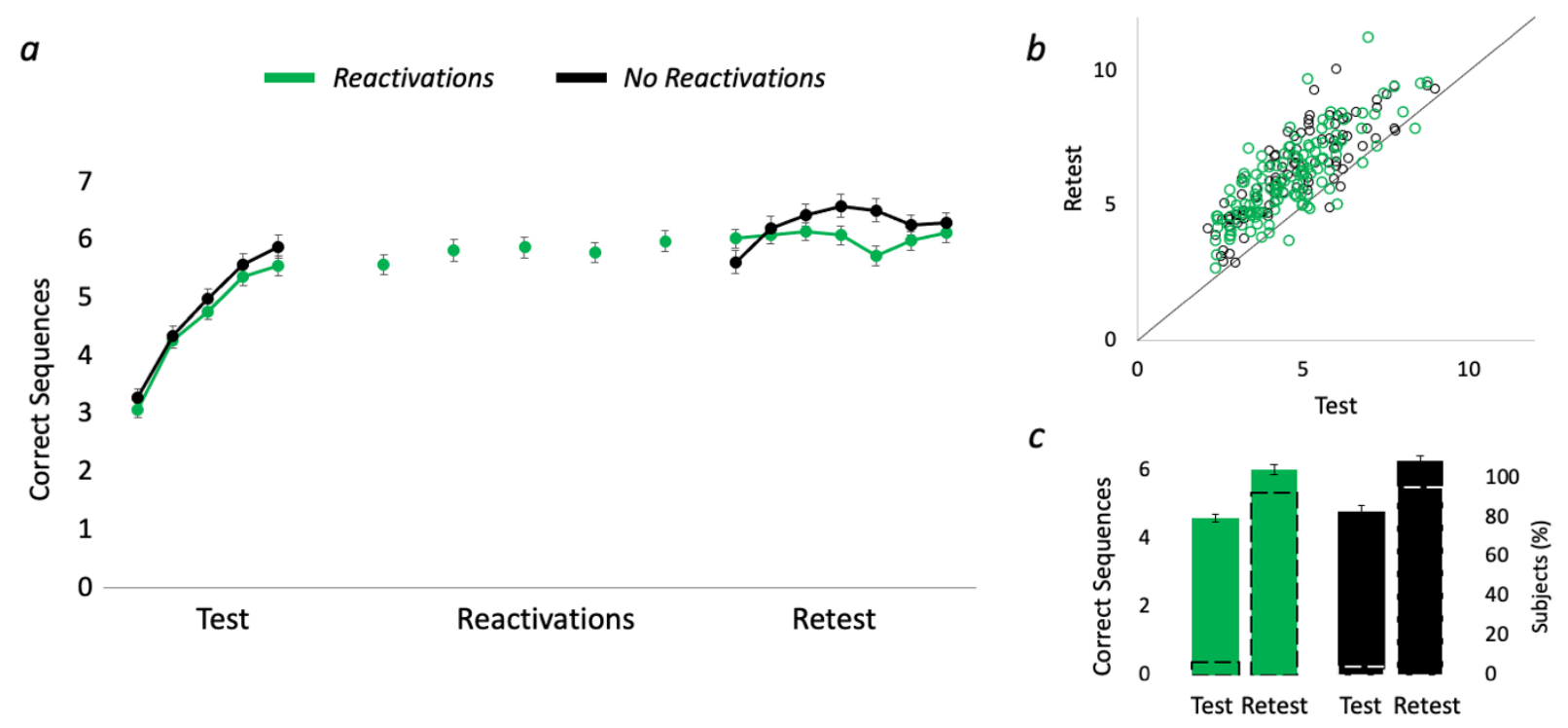

Figure 3: Micro-timescale reactivations do not induce learning. (a) Single-trial performance (Reactivations group in yellow and NoReactivation group in black). (b) Test versus retest single-subject performance presented in a scatterplot along a unit slope line $(\mathrm{y}=\mathrm{x})$ where each point reflects a participant $(11,16,30)$. Data accumulating above the unit line reflect subjects who improved from test to retest, expressing learning gains, while data points below the line indicate degraded retest performance. (c) Colored bars reflect the mean performance in test and retest sessions (corresponding to the left y-axis), dashed black bars (corresponding to the right $y$-axis) reflect the percentage of participants on each side of the unit slope line in b. Error bars represent SEM. 


\section{Discussion}

In a set of crowdsourced experiments, with data collected from hundreds of participants, this study examined whether reconsolidation of motor skill memories operates at a micro-timescale level. To address this question, two main behavioral outcomes of memory reconsolidation were tested: memory interference and enhancement. Based on multiple studies reporting modified effects of memory interference if a new memory is presented during reconsolidation time windows $(8,16,28)$, Experiment 1 tested whether interference with reactivated skill memory is enhanced immediately following encoding. However, results showed that the effect of interference was comparable regardless of whether the new memory was reactivated or not. In addition, since memory reactivation was recently reported to induce learning in motor skills (17, 18), Experiment 2 tested whether uninterrupted reactivations following early skill learning, enhance performance. Results showed that these reactivations did not enhance skill performance, with performance at retest comparable to the NoReactivations group who did not undergo reactivations between test and retest. In sum, these results show that memory reconsolidation effects reported in both human and animal studies, do not apply at the micro-timescale level, and thus do not increase the susceptibility of memories to modulations in early stages of learning. Interestingly, rapid consolidation was reported to occur in early stages of motor skill learning, during rest periods between trials, lasting only 10 seconds (26). Moreover, a recent study suggested that micro-timescale consolidation might be induced by fast neural replay occurring during wakeful rest periods between trials (31). Even though consolidation is evident at the micro-timescale, the current study did not find evidence for an analogous form of rapid memory reconsolidation occurring at the micro-timescale level. The reason for this might stem from the state of the memory trace itself. Memory consolidation transforms unstable newly acquired memories to a stable state, in which they will be less prone to modifications, while memory reconsolidation occurs when the memory trace is already in a stable state, transforming the memory trace back to an unstable state. Thus, since consolidation affects unstable memories, it may be effective for newly acquired skills at early stages of learning, but reconsolidation, operating on stable memory traces, might not be effective in early stages of learning, and therefore does not operate successfully in a rapid form at the micro-timescale level. 
When a second skill memory was presented during the rapid reconsolidation time windows in Experiment 1, performance of both memories gradually converged to a common level, which was better than the starting level of new memory, but worse than the starting level of the original memory. This notion might suggest that, in early stages of learning, when the memory is still flexible enough, two memories can be acquired simultaneously, with a small detriment in performance of the original memory. Moreover, the convergence of performance towards a similar level of skill execution might imply that these memories are stored in a shared manner in the brain as a single merged memory trace, consistent with the concept of neural engrams (32), which were shown to overlap when learning two linked memories (33).

These findings provide additional evidence for the long-known discussion on the fundamental dissociation between consolidation and reconsolidation (34). A number of studies have tested the molecular differences between the two, with some studies reporting similarities at the molecular level of the underlying mechanisms $(3,35)$, and some studies reporting differences (for example (36-39)). In line with these studies, the results of the current study point to a dissociation between consolidation and reconsolidation, with a behavioral separation between consolidation, that indeed occurs at a rapid form within minutes $(26,40)$, and reconsolidation that does not operate in such a micro-timescale.

In sum, the findings of the current study provide robust evidence that contrary to the regular timescales of memory reconsolidation, interference (experiment 1) and learning (experiment 2) are not enhanced within a rapid reconsolidation time window. Thus, findings of both experiments converge to suggest that reconsolidation does not operate at a micro-timescale level. Revealing the boundaries and time frames of memory reconsolidation can have implications on future strategies geared to modulate learning and memory. 


\section{References}

1. A. Nettersheim, M. Hallschmid, J. Born, S. Diekelmann, The role of sleep in motor sequence consolidation: stabilization rather than enhancement. J. Neurosci. 35, 66966702 (2015).

2. J. L. McGaugh, Memory--a Century of Consolidation. Science (80-. ). 287, 248 LP - 251 (2000).

3. K. Nader, G. E. Schafe, J. E. Le Doux, Fear memories require protein synthesis in the amygdala for reconsolidation after retrieval. Nature 406, 722-726 (2000).

4. A. Suzuki, et al., Memory Reconsolidation and Extinction Have Distinct Temporal and Biochemical Signatures. J. Neurosci. 24, 4787-4795 (2004).

5. J. L. C. Lee, Memory reconsolidation mediates the strengthening of memories by additional learning. Nat. Neurosci. 11, 1264-1266 (2008).

6. N. Herz, et al., Neuromodulation of Visual Cortex Reduces the Intensity of Intrusive Memories. Cereb. Cortex (2021) https:/doi.org/10.1093/cercor/bhab217.

7. N. Censor, S. G. Horovitz, L. G. Cohen, Interference with Existing Memories Alters Offline Intrinsic Functional Brain Connectivity. Neuron 81, 69-76 (2014).

8. M. P. Walker, T. Brakefield, J. A. Hobson, Dissociable stages of human memory consolidation and reconsolidation. Nature 425, 616-620 (2003).

9. E. Gabitov, et al., Re-stepping into the same river: competition problem rather than a reconsolidation failure in an established motor skill. Sci. Rep. 7, 9406 (2017).

10. D. Shmuel, et al., Early Visual Cortex Stimulation Modifies Well-Consolidated Perceptual Gains. Cereb. Cortex 31, 138-146 (2021).

11. R. Amar-Halpert, R. Laor-Maayany, S. Nemni, J. D. Rosenblatt, N. Censor, Memory reactivation improves visual perception. Nat. Neurosci. 20, 1325 (2017).

12. J. Herszage, N. Censor, Modulation of learning and memory: A shared framework for interference and generalization. Neuroscience (2018).

13. Y. Dudai, Reconsolidation: the advantage of being refocused. Curr. Opin. Neurobiol. 16, 174-178 (2006).

14. J. W. B. Elsey, V. A. Van Ast, M. Kindt, Human memory reconsolidation: A guiding framework and critical review of the evidence. Psychol. Bull. 144, 797 (2018).

15. D. Schiller, et al., Preventing the return of fear in humans using reconsolidation update 
mechanisms. Nature 463, 49-53 (2010).

16. J. Herszage, N. Censor, Memory Reactivation Enables Long-Term Prevention of Interference. Curr. Biol. 27, 1529-1534.e2 (2017).

17. J. Herszage, H. Sharon, N. Censor, Reactivation-induced motor skill learning. Proc. Natl. Acad. Sci. 118 (2021).

18. N. F. Wymbs, A. J. Bastian, P. A. Celnik, Motor skills are strengthened through reconsolidation. Curr. Biol. 26, 338-343 (2016).

19. R. Stickgold, M. P. Walker, Memory consolidation and reconsolidation: what is the role of sleep? Trends Neurosci. 28, 408-415 (2005).

20. S. Duvarci, K. Nader, Characterization of fear memory reconsolidation. J. Neurosci. 24, 9269-9275 (2004).

21. M.-H. Monfils, K. K. Cowansage, E. Klann, J. E. LeDoux, Extinction-reconsolidation boundaries: key to persistent attenuation of fear memories. Science (80-. ). 324, 951-955 (2009).

22. T. Brashers-Krug, R. Shadmehr, E. Bizzi, Consolidation in human motor memory. Nature 382, 252-255 (1996).

23. D. Z. Press, M. D. Casement, A. Pascual-Leone, E. M. Robertson, The time course of offline motor sequence learning. Cogn. Brain Res. 25, 375-378 (2005).

24. M. P. Walker, R. Stickgold, Sleep-dependent learning and memory consolidation. Neuron 44, 121-133 (2004).

25. D. Lugassy, J. Herszage, R. Pilo, T. Brosh, N. Censor, Consolidation of complex motor skill learning: evidence for a delayed offline process. Sleep 41, zsy123 (2018).

26. M. Bönstrup, et al., A rapid form of offline consolidation in skill learning. Curr. Biol. 29, 1346-1351 (2019).

27. A. Karni, et al., Functional MRI evidence for adult motor cortex plasticity during motor skill learning. Nature 377, 155-8 (1995).

28. T. T. de Beukelaar, D. G. Woolley, N. Wenderoth, Gone for 60 seconds: Reactivation length determines motor memory degradation during reconsolidation. Cortex 59, 138-145 (2014).

29. A. Karni, et al., The acquisition of skilled motor performance: fast and slow experiencedriven changes in primary motor cortex. Proc. Natl. Acad. Sci. U. S. A. 95, 861-8 (1998). 
30. S. Gelstein, et al., Human tears contain a chemosignal. Science (80-. ). 331, 226-230 (2011).

31. E. R. Buch, L. Claudino, R. Quentin, M. Bönstrup, L. G. Cohen, Consolidation of human skill linked to waking hippocampo-neocortical replay. Cell Rep. 35, 109193 (2021).

32. S. A. Josselyn, S. Köhler, P. W. Frankland, Finding the engram. Nat. Rev. Neurosci. 16, 521-534 (2015).

33. G. Kastellakis, A. J. Silva, P. Poirazi, Linking Memories across Time via Neuronal and Dendritic Overlaps in Model Neurons with Active Dendrites. Cell Rep. 17, 1491-1504 (2016).

34. Y. Dudai, The Restless Engram : Consolidations Never End. Annu. Rev. Neurosci. 35, 227-247 (2012).

35. J. W. Bang, et al., Consolidation and reconsolidation share behavioural and neurochemical mechanisms. Nat. Hum. Behav. 2, 507-513 (2018).

36. J. L. C. Lee, B. J. Everitt, K. L. Thomas, Independent cellular processes for hippocampal memory consolidation and reconsolidation. Science (80-. ). 304, 839-843 (2004).

37. L. S. J. von Hertzen, K. P. Giese, Memory reconsolidation engages only a subset of immediate-early genes induced during consolidation. J. Neurosci. 25, 1935-1942 (2005).

38. Y. Li, et al., Learning and reconsolidation implicate different synaptic mechanisms. Proc. Natl. Acad. Sci. 110, 4798-4803 (2013).

39. N. Censor, E. R. Buch, K. Nader, L. G. Cohen, Altered human memory modification in the presence of normal consolidation. Cereb. Cortex 26, 3828-3837 (2016).

40. M. Bönstrup, I. Iturrate, M. N. Hebart, N. Censor, L. G. Cohen, Mechanisms of offline motor learning at a microscale of seconds in large-scale crowdsourced data. NPJ Sci. Learn. 5, 7 (2020). 